\title{
Detailed chemistry LES/CMC simulation of a swirling ethanol spray flame approaching blow-off
}

\author{
A. Giusti*, E. Mastorakos \\ Department of Engineering, University of Cambridge, Trumpington Street, Cambridge \\ CB2 1PZ, UK
}

\begin{abstract}
A swirling ethanol spray flame in conditions close to blow-off has been simulated using Large Eddy Simulation (LES) and the Conditional Moment Closure (CMC) combustion model aiming to further validate the capability of the LES/CMC approach to capture local extinctions in turbulent spray flames. A detailed chemical mechanism was used and a transport equation of the mixture fraction sub-grid variance, with spray interaction terms included, was solved. Numerical results are in good agreement with the experiment in terms of both instantaneous and mean flame shape and droplet velocity and size. Local extinctions were detected in the region around the bluffbody, resulting in a fluctuating lift-off of the flame there, and the probability density function of the lift-off height was in very good agreement with the experiment, suggesting that the degree of local extinction is captured quantitatively. Analysis of the CMC equation suggested that local extinction was influenced by both transport in physical space and high scalar dissipation rate. The modelling of the latter needs development in areas where the
\end{abstract}

\footnotetext{
${ }^{*}$ Corresponding author

email address: ag813@cam.ac.uk 
spray evaporation is strong enough to increase significantly the sub-grid mixture fraction fluctuations and their small-scale gradients, possibly leading to deviations from the present usual approach of relating the sub-grid scalar dissipation to the sub-grid mixture fraction variance.

Keywords: Conditional Moment Closure, Large Eddy Simulation, Swirling spray flame, Localised extinction, Lift-off

\section{Introduction}

Swirling spray flames are of great interest for many combustion systems. At adverse conditions, finite rate chemistry effects become important leading to the presence of local extinctions and eventually blow-off, a phenomenon that has been recently studied in laboratory-scale flames with various liquid fuels [1]. Our simulation capability of extinction phenomena is not fully validated yet, not least because capturing the local extinction and its evolution into a global blow-off has not been demonstrated extensively with current generation turbulent combustion models. Some success in predicting the local extinction of gaseous non-premixed flames has already been demonstrated with Large Eddy Simulation and the CMC, Eulerian transported PDF, and flamelet-progress variable models [4] 8 . In particular, the LES/CMC approach applied to the simulation of the swirling non-premixed methane flame of Ref. [1], predicted with reasonable accuracy both the degree of local extinction, described in terms of lift-off height statistics [8], and the experimental blow-off limits for a wide range of air flow bulk velocities [9]. However, similar capability for spray combustion has not been demonstrated yet, except for two LES/CMC attempts [10, 11]. In both these studies, the 
simulations were performed using a modified one-step chemistry model [12], that was tuned so as to give a reasonable prediction of the extinction strain rate of a laminar non-premixed flamelet. However, since the process leading to local extinctions is affected by the temporal fluctuations of the scalar dissipation, but also by transport effects [6, 13, 14], a more reliable simulation of the extinction and re-ignition phenomena needs recourse to detailed chemical mechanisms.

Spray flames are generally characterized by a strong coupling with the evaporation [15], which affects the spatial distribution of the fuel, possibly increasing the sub-grid scale fluctuations of the mixture fraction and the small-scale gradients. There is evidence from DNS [16, 17] that the evaporation could have an important effect on the sub-grid mixture fraction variance. Therefore, in the context of the LES/CMC approach, detailed attention should be devoted to the modelling of the sub-grid scale mixture fraction variance since this quantity is directly involved in the computation of both the filtered probability density function and the sub-grid scale scalar dissipation rate, which in turn affects the extinction behaviour. Due to lack of consensus on their modelling, spray effects on the mixture fraction variance field are often neglected and the variance is simply modelled through the same closure used for gaseous flames [18, 19]. Some attempts to include spray evaporation effects in the computation of the sub-grid mixture fraction variance were done in Refs. [10, 11] where a formulation based on the equilibrium model proposed by Pera et al. [17] was used. However, as pointed out in [17], such model presents some difficulties in predicting a proper level of variance even for simple homogeneous flows suggesting the use of a transport 
equation to improve the prediction capability.

In this work, an ethanol spray flame [2] at conditions close to blow-off, where a large degree of local extinction is present, is simulated using the LES/CMC approach and a detailed chemical mechanism. Furthermore, in order to better evaluate the effect of evaporation on the sub-grid scale fluctuations of the mixture fraction, an equation for the sub-grid mixture fraction variance with spray terms included was solved. The objectives are: (i) to further validate the LES/CMC method in predicting local extinction in turbulent spray flames, (ii) to give more insight into the flame structure and the mechanisms affecting local extinction. The numerical method is presented next, followed by results that focus on the local extinction behaviour and the main conclusions.

\section{Numerical method}

\subsection{Mathematical models}

The simulations are based on an Eulerian-Lagrangian approach for dilute sprays where the evolution of the gas phase is solved using Large Eddy Simulation (LES) and the combustion is modelled through the Conditional Moment Closure (CMC) model [10, 20,24]. The CMC equation for a condi-

tionally filtered reacting scalar, $Q_{\alpha}=\widetilde{Y_{\alpha} \mid \eta}$, can be written in the following 
form [10, 24]:

$$
\begin{aligned}
\frac{\partial Q_{\alpha}}{\partial t}+\underbrace{\frac{\partial}{\partial x_{i}}\left(Q_{\alpha} \widetilde{u_{i} \mid \eta}\right)}_{\text {convection }}-\underbrace{\left.Q_{\alpha} \frac{\partial}{\partial x_{i}} \widetilde{\left(u_{i} \mid \eta\right.}\right)}_{\text {dilatation }}=e_{\alpha}+\widetilde{N \mid \eta} \frac{\partial^{2} Q_{\alpha}}{\partial \eta^{2}} \\
+\widetilde{\omega_{\alpha} \mid \eta}+\delta_{\alpha, f} \widetilde{\Pi \mid \eta}-\left(Q_{\alpha}+(1-\eta) \frac{\partial Q_{\alpha}}{\partial \eta}\right) \widetilde{\Pi \mid \eta}
\end{aligned}
$$

where the term representing the transport in physical space was decomposed into a convection and a dilatation term, following the unstructured CMC implementation adopted here [8, 22]. The unconditional filtered quantities are obtained from the respective conditional values by means of a Filtered probability Density Function (FDF), $\widetilde{P}(\eta)$, which was assumed to have a $\beta$ function shape computed from the resolved mixture fraction, $\widetilde{\xi}$, and the subgrid scale mixture fraction variance, $\widetilde{\xi^{\prime \prime 2}}$. In Eq. 1. $e_{\alpha}$ represents the contribution from the sub-grid scales which was modelled using the typical gradient assumption, neglecting the contribution involving the sub-grid conditional joint fluctuations of the droplet evaporation rate and species [10, 19]. For the conditional velocity, $\widetilde{u_{i} \mid \eta}=\widetilde{u_{i}}$ was assumed. The conditional scalar dissipation rate, $\widetilde{N \mid \eta}$, was closed with the Amplitude Mapping Closure (AMC) model [25, 26]: $\widetilde{N \mid \eta}=N_{0} G(\eta)$, with $G(\eta)=\exp \left(-2\left[\operatorname{erf}^{-1}(2 \eta-1)\right]^{2}\right)$ and $N_{0}=\widetilde{N} / \int_{0}^{1} G(\eta) \widetilde{P}(\eta) d \eta$. The filtered scalar dissipation rate $\tilde{N}$ was computed considering both resolved and sub-grid contributions:

$$
\widetilde{N}=\widetilde{N}_{\text {res }}+\widetilde{N}_{\text {sgs }}=\underbrace{D \frac{\partial \widetilde{\xi}}{\partial x_{i}} \frac{\partial \widetilde{\xi}}{\partial x_{i}}}_{\text {resolved }}+\underbrace{\frac{1}{2} C_{N} \frac{\mu_{t}}{\Delta^{2} \bar{\rho}} \widetilde{\xi^{\prime \prime 2}}}_{\text {sub-grid scale }}
$$


where $D$ is the molecular diffusivity $(D=\mu /(\bar{\rho} S c)$ with $S c=0.7), \mu_{t}$ is the sub-grid scale viscosity and $C_{N}$ is a model constant taken equal to 42.0. This value, obtained through calibration against scalar dissipation rate measurements in the Sandia D flame [6], has been recently applied to simulations of both gaseous non-premixed [8, 9, 22] and spray flames [10, 11] giving reasonable predictions of the local extinction behaviour compared to experiments. The term $\widetilde{\Pi \mid \eta}$, representing conditional source terms due to spray evaporation, was modelled as discussed in [10]. Limiters were applied to this source term for the values of $\eta$ characterized by a very low probability, in order to avoid numerical instabilities [27]. First order closure was used for the chemical source term $\widetilde{\omega_{\alpha} \mid \eta}$. A similar equation (not reported here, see for example Ref. [10]) without chemical source term, was solved for the total enthalpy.

A detailed chemical mechanism [28] with 57 species and 383 reversible reactions was used. The extinction behaviour of this mechanism was evaluated in preliminary computations performed with the so called 0D-CMC approach (i.e., where the CMC equations are solved without terms representing transport in physical space and spray source terms and with a prescribed $N_{0}$ ); this is similar to a flamelet solution with unity Lewis number and a given distribution of the scalar dissipation rate. Although the local flame structure in a $\mathrm{CMC}$ computation is the result of the local solution of the $\mathrm{CMC}$ equations (see Eq. 1), which is dependent on transient effects, the local scalar dissipation rate, and the transport terms, the 0D-CMC solution will be briefly analysed as a useful reference for the remainder of the discussion. Figure 1 shows the flame structure in $\eta$-space for $N_{0}=1201 / \mathrm{s}$. The fuel is consumed 


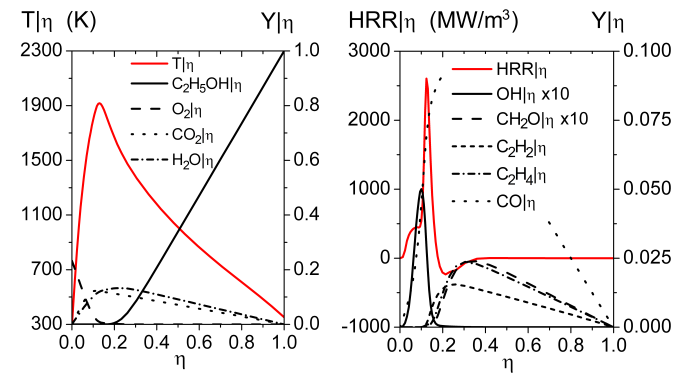

Figure 1: Temperature and species mass fractions from a 0D-CMC computation $\left(N_{0}=1201 / \mathrm{s}\right)$.

before reaching the stoichiometric mixture fraction $\left(\eta_{s t} \approx 0.1\right)$ through pyrolysis that leads to the production of intermediate species (such as $\mathrm{C}_{2} \mathrm{H}_{2}$ and $\mathrm{C}_{2} \mathrm{H}_{4}$ ) and to a negative heat release rate. The $\mathrm{OH}$ is finite in a thin region around stoichiometry, disappearing quickly at rich mixture fractions $(\eta>0.2)$. The critical scalar dissipation rate $N_{0, c r}$, i.e. the maximum value of $N_{0}$ above which no steady-state burning solution exists, was $3671 / \mathrm{s}$ (with the corresponding critical value at the stoichiometric mixture fraction equal to $\left.N_{s t, c r} \approx 711 / \mathrm{s}\right)$.

Concerning the flow field, the Favre-filtered equations for the conservation of mass and momentum with the low-Mach number assumption were solved together with an equation for the filtered mixture fraction [10]. The sub-grid scale stress tensor was closed with the Vreman model [29] able to give a vanishing turbulent viscosity close to solid walls. The sub-grid scale mixture fraction was computed by a transport equation with spray source 
terms included [17, 23]:

$$
\begin{aligned}
\frac{\partial \bar{\rho} \widetilde{\xi^{\prime \prime 2}}}{\partial t}+\frac{\partial \bar{\rho} \widetilde{u}_{i} \widetilde{\xi^{\prime \prime 2}}}{\partial x_{i}} & =\frac{\partial}{\partial x_{i}}\left(\bar{\rho}\left(D+D_{t}\right) \frac{\partial \widetilde{\xi^{\prime \prime 2}}}{\partial x_{i}}\right)-2 \bar{\rho} \widetilde{N} \\
& +2 \bar{\rho}\left(D+D_{t}\right) \frac{\partial \widetilde{\xi}}{\partial x_{i}} \frac{\partial \widetilde{\xi}}{\partial x_{i}}+\underbrace{2 \bar{\rho}(\widetilde{\xi \Pi}-\widetilde{\xi})}_{\bar{\rho} W^{+}}-\underbrace{\bar{\rho}\left(\widetilde{\xi^{2} \Pi}-\widetilde{\xi} \widetilde{\Pi}\right)}_{\bar{\rho} W^{-}}
\end{aligned}
$$

where $D_{t}=\mu_{t} /\left(\bar{\rho} S c_{t}\right)$ is the turbulent diffusivity $\left(S c_{t}=0.4\right.$ was assumed [10] $)$. The scalar dissipation rate $\widetilde{N}$ was closed as in Eq. 2 and the term $\widetilde{\xi \Pi}$ was modelled as discussed in [10]. A similar closure was adopted here also for the term $\widetilde{\xi^{2} \Pi}$ resulting in $\widetilde{\xi^{2} \Pi}=\bar{\xi}_{s}^{2} \widetilde{\Pi}$, where $\bar{\xi}_{s}$ is the saturation mixture fraction [10]. It should be noted that the evaporation could also affect the shape of both the FDF and the conditional scalar dissipation rate [27]. Here it is assumed that the models for FDF and $\widetilde{N \mid \eta}$ do not change with evaporation and the effects of the spray are represented only through the sub-grid scale mixture fraction variance. Further models for the possible influence of the evaporation on $\widetilde{P}(\eta)$ and $\widetilde{N \mid \eta}$ must be developed.

The dilute spray approximation was adopted for the Lagrangian tracking of spray parcels, while the Abramzon and Sirignano model [30] was used for evaporation. No secondary breakup model was needed due to the low Weber number [2].

\subsection{Solution strategy}

The LES unstructured code PRECISE-UNS [31] was used for the flowfield solution whereas the CMC equations were solved using a superimposed unstructured CMC code [8, 22]. The coupling between the two solvers, 
achieved through the density and temperature, follows the implementation discussed in [22] with additional features due to the spray. Following common practice, the CMC equations were solved in a mesh coarser than the one used for the flow making necessary the transfer of quantities computed at the LES resolution to the CMC grid [22, 23]. Both the scalar dissipation rate and spray source terms at the $\mathrm{CMC}$ resolution were computed by means of a FDF-weighted average of the respective values at the LES level.

Operator splitting was used for the solution of the CMC equations with the chemical source term treated by the VODPK implicit solver [32]. Firstorder upwind scheme was used for convective terms whereas diffusion terms were discretised with second-order schemes and a first-order scheme was used for time discretisation. Concerning the LES, second-order accurate schemes were used for spatial discretisation together with a second-order implicit backward scheme for time derivatives.

The investigated burner [2] consists of a pressure atomizer (hollow-cone with nominal spray angle $60^{\circ}$ ), fitted in a bluff-body holder of diameter $D=25 \mathrm{~mm}$, and a square section enclosure open to the atmosphere at the outlet. The swirling air flow is supplied through the annular duct surrounding the bluff body. The experimental condition E1S1, characterized by an air flow bulk velocity $U_{b}$ equal to $17.1 \mathrm{~m} / \mathrm{s}$ ( $79.2 \%$ of the blow-off velocity) and a fuel mass flow rate of $0.27 \mathrm{~g} / \mathrm{s}$, was considered. Measurements include Mie scattering from the spray droplets, mean axial droplet velocity and Sauter Mean Diameter (SMD), $\mathrm{OH}^{*}$ chemiluminescence that provides qualitative information of the heat release, and qualitative OH-PLIF.

The air inlet in the numerical domain was located immediately down- 
stream of the swirler [11. A hexahedral mesh of about 5 million cells and with a minimum grid size of $0.2 \mathrm{~mm}$ in the vicinity of the bluff body was used for the LES, whereas a hexahedral mesh of 45,000 cells was used for the $\mathrm{CMC}$ equations. This mesh was selectively refined in the flame region with a maximum grid size lower than $1.5 \mathrm{~mm}$ in the all flame region (up to $40.0 \mathrm{~mm}$ above the bluff body in the axial direction and $20.0 \mathrm{~mm}$ along the radial direction) and a resolution of $0.5 \mathrm{~mm}$ immediately downstream of the bluff-body edge. 51 nodes clustered around $\eta_{s t}$ were used to discretise the mixture fraction space, with the lower and upper boundaries located respectively at $\eta=0.0$ and $\eta=1.0$. As also discussed in [18, 33], the maximum value of the mixture fraction could have an important effect on the solution. Here, the boundary was located at $\eta=1.0$ as the maximum possible physical value, corresponding to a boiling droplet, letting the FDF represent the actual properties of the mixture. This is also consistent with the use of the AMC model for the conditional scalar dissipation rate which is defined in the same range of $\eta$.

Uniform axial and swirl velocities were imposed at the inlet boundary, a constant pressure condition was used at the outlet, and all solid surfaces were modelled as adiabatic walls with the no-slip condition. As far as conditional quantities are concerned, the inert mixing solution was imposed at the inlet whereas both the walls and the outlet were modelled with a zero-gradient condition. $\eta=0.0$ corresponds to pure air at ambient temperature whereas at $\eta=1.0$ pure vaporised fuel at the boiling temperature was imposed [10, 27]. A time step equal to $2 \mu \mathrm{s}$ was used for both the LES and CMC solvers.

According to the experiment [2], the injected droplets show a dispersion 


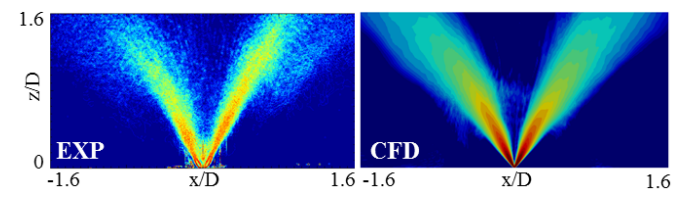

Figure 2: Mean Mie scattering image from the experiment [2] (left) and the mean Mie equivalent quantity from LES (right) in log-scale.
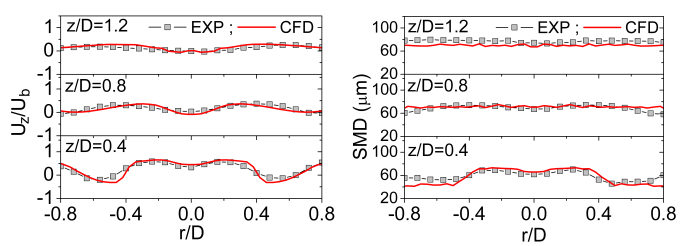

Figure 3: Numerical results and experimental measurements 2] of droplet mean axial velocity and Sauter Mean Diameter (SMD).

around the nominal cone angle. To reproduce this behaviour, the spray injection was modelled by assigning a mean injection angle, equal to the nominal spray angle, and a random component added to it sampled from a truncated normal distribution with a standard deviation $\sigma_{\theta}=6^{\circ}$. Diameters were computed from a Rosin-Rammler distribution with Sauter Mean Diameter equal to $70 \mu \mathrm{m}$ and dispersion parameter $q=4.0$. The magnitude of the injection velocity, assumed to be equal for all the injected parcels, was selected in order to have a good agreement with experiments at the first measurement location. The simulation was performed on $1282.6 \mathrm{GHz}$ processors with $4 \mathrm{~GB}$ of RAM per processor. The computation of $1 \mathrm{~ms}$ of physical time requires almost $36 \mathrm{~h}$.

\section{Results and discussion}

Figure 2 compares the experimental mean Mie scattering image [2] with a numerical equivalent quantity (proportional to the sum of the surface of 


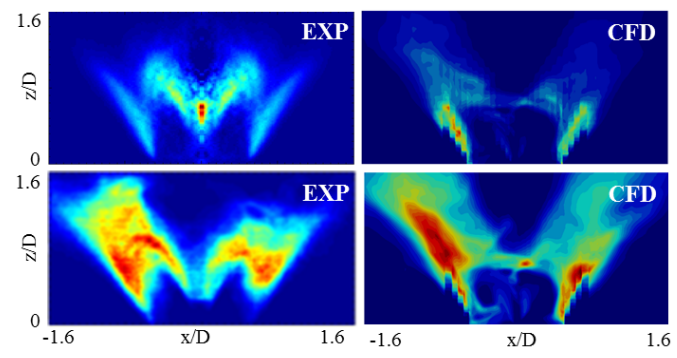

Figure 4: Top row: experimental inverse Abel-transformed time-averaged $\mathrm{OH}^{*}$ 2] (left), LES mean heat release rate (right). Bottom row: mean OH-PLIF from the experiment [2] (left), mean $\mathrm{OH}$ mass fraction from LES (right).

droplets crossing each cell [11]) and allows us to analyse the location and dispersion of the spray. Furthermore, in Fig. 3 the SMD and axial velocity at different axial distances, $z$, from the bluff body are given (numerical results were obtained through an azimuthal average of 10 spray realizations sampled with a sampling frequency of $1 \mathrm{kHz}$ ). The location and spreading of the spray and the droplet diameter and velocity appear quite well predicted, especially close to the bluff body therefore giving an overall assessment of the reliability of the adopted approach and boundary conditions in reproducing the main features of the spray.

In Fig. 4 the mean heat release rate (HRR) and $\mathrm{OH}$ mass fraction from LES are compared with the inverse Abel-transformed time-averaged $\mathrm{OH}^{*}$ and mean OH-PLIF signal from the experiment 2], which can be used to get information regarding the location and the shape of the reacting regions. It is possible to distinguish two flame regions, an outer flame brush located along the shear layer between the annular air and the recirculation zone, and an inner flame located inside the spray cone. The numerical results are in relatively good agreement with the experiment, with both the location and 
the extent of the mean reacting region quite well predicted. The absence of $\mathrm{OH}$ inside the hollow cone spray and between the spray and the annular air is consistent with the experiment. Although in the present experiment the OH-PLIF and $\mathrm{OH}^{*}$ chemiluminescence cannot be considered quantitative, the results are considered satisfactory and capture the main characteristics of the flame. However, the relative level of the HRR and mean $\mathrm{OH}$ in the inner compared to the outer flame regions is under-predicted in the simulation. As discussed in the following, this region is characterized by strong contributions of the evaporating spray to the sub-grid variance, and since the sub-grid dissipation model has not been adapted to account for the spray, it may be that the scalar dissipation is not captured properly in the inner flame branch that is aligned with the spray.

Figure 5 shows some flow field quantities and mass fractions of selected species in a $z-r$ plane at a given time instant, whereas in Fig. 6 an instantaneous stoichiometric mixture fraction iso-surface is shown. The region above the bluff body is characterized by a rich mixture due to the large amount of fuel released through evaporation. The $\mathrm{CH}_{2} \mathrm{O}$ mass fraction is generally high in this region [3] and the formation of intermediate species (such as $\mathrm{C}_{2} \mathrm{H}_{2}$ and $\mathrm{C}_{2} \mathrm{H}_{4}$ ) through pyrolysis is observed leading to negative values of HRR (as also indicated by the the flame structure of Fig. 11). A considerable amount of $\mathrm{CO}$ and $\mathrm{H}_{2}$ is also produced in rich $\widetilde{\xi}$. All the intermediate species are eventually consumed in the thin reaction zone and only traces are present in the lean regions. The evaporation gives an important contribution to the mixture fraction variance, as highlighted by the peaks of $\widetilde{\xi^{\prime \prime 2}}$ along the spray path in Fig. 5. The large variance increases $\widetilde{N}_{s g s}$, which becomes dominant 


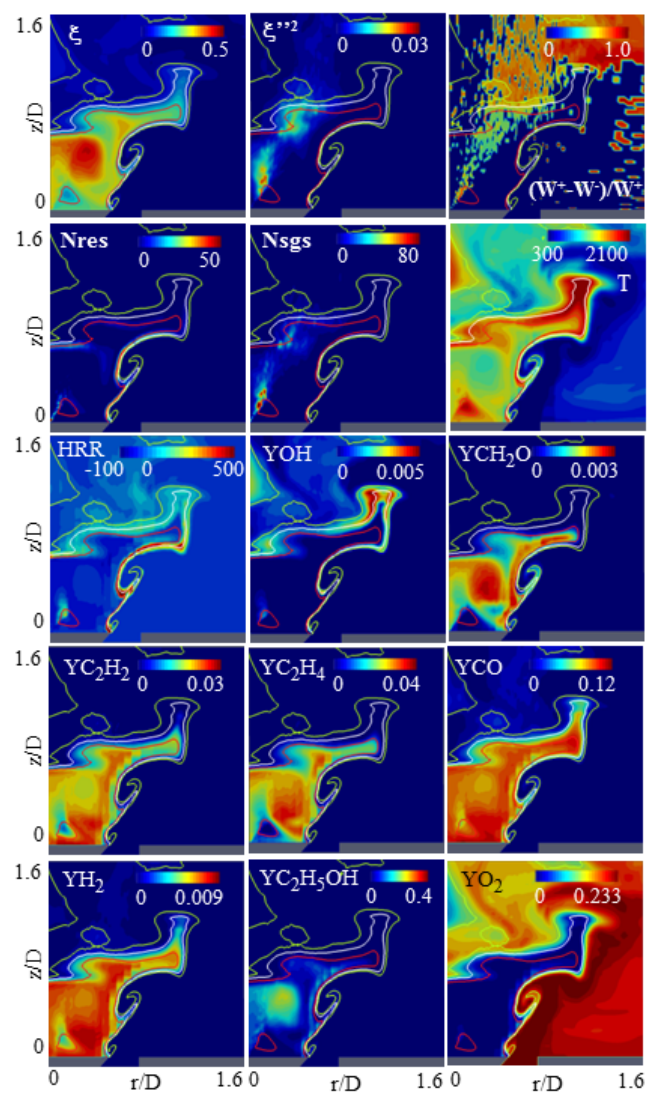

Figure 5: Instantaneous unconditional quantities in a $z-r$ cross section. White line: stoichiometric mixture fraction; red line: $\eta=0.2$; green line: $\eta=0.05$.

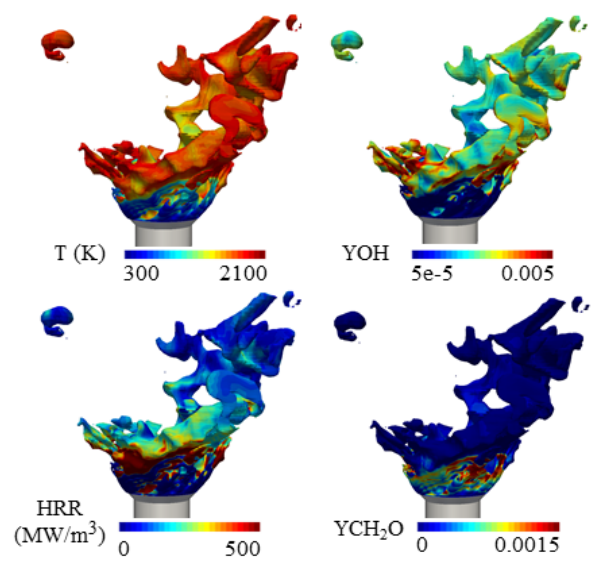

Figure 6: Stoichiometric mixture fraction iso-surface coloured by the indicated quantity. 
over $\widetilde{N}_{\text {res }}$. According to Ref. [17], $\bar{\rho} W^{-}$should be negligible compared to the other terms, however, as shown in Fig. 5 through the ratio $\left(W^{+}-W^{-}\right) / W^{+}$ (please note that in the cells without droplets this quantity was set equal to zero), this term is of the same order of magnitude as $\bar{\rho} W^{+}$and therefore cannot be neglected. The relatively high scalar dissipation rate together with a wider shape of the FDF function lead to lower values of temperature, which in turn affects the droplet evaporation. This also explains the lower level of $\mathrm{OH}$ mass fraction observed in the stoichiometric region lying along the spray path as well as the underestimation of the mean $\mathrm{OH}$ level in that region. By comparison with Fig. 2, some of the droplets cross the flame front. Residual evaporation in the lean region of the flame (e.g. $r / D \approx 0.7, z / D \approx 1.0$ ) can sustain the reaction leading to the formation of small amounts of $\mathrm{OH}$ that seem to have a wider spatial distribution (smaller gradients), following the very low $\widetilde{N}$ in that region. Due to the overall lean equivalence ratio of the combustor, the opposing (recirculating) flow has large amounts of oxygen, associated with mixing of the hot products of the flame with fresh reactants, that then act as the oxidiser of the inner flame.

The stoichiometric mixture fraction iso-surface (Fig. 6) is generally attached to the bluff body edge. However, the reaction zone in some of the locations appears lifted-off, as revealed by the negligible values of $\mathrm{OH}$ together with low temperature and negligible HRR, clearly indicating the presence of local extinctions along the $\xi_{s t}$ iso-surface. This is consistent with the experiment [2], where the OH-PLIF measurements revealed a large amount of local extinctions as shown in Fig. 7, where a $\mathrm{OH}$ mass fraction snapshot from the LES is directly compared with an instantaneous OH-PLIF image from the 


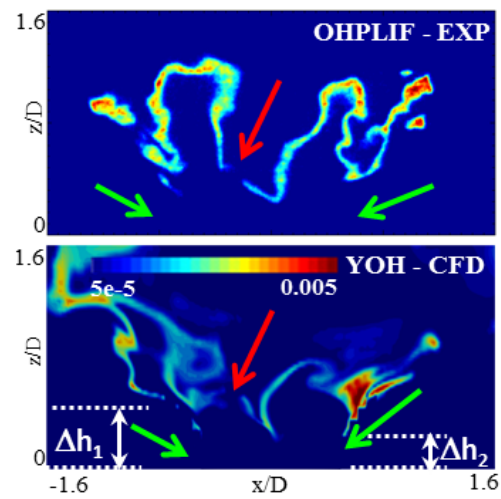

Figure 7: Comparison between an instantaneous OH-PLIF image from the experiment [2] and instantaneous $\mathrm{OH}$ mass fraction from LES. Green arrows: local extinction along the outer flame brush; red arrow: local extinction in the inner flame region.

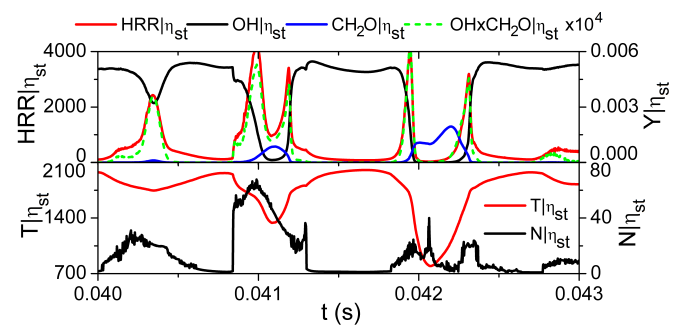

Figure 8: Time evolution of conditional quantities at the stoichiometric mixture fraction in a $\mathrm{CMC}$ cell located at $r / D=0.6, z / D=0.4$.

experiment [2]. In more detail, Fig. 7 shows the presence of "holes" along the $\mathrm{OH}$-sheet, attributed to local extinctions, on both inner and outer flame zones. The OH-containing zones are relatively thin, characteristic of nonpremixed flames. The first emergence of $\mathrm{OH}$ downstream of the bluff body corner is treated as a lifted flame in that region, and the statistics of the lift-off height marked in Fig. 7 are discussed later.

The local extinction behaviour is now analysed considering the solution in $\eta$-space. Figures 8 and 9 show the time evolution of selected conditional quantities in a location along the outer flame brush. As indicated by the 


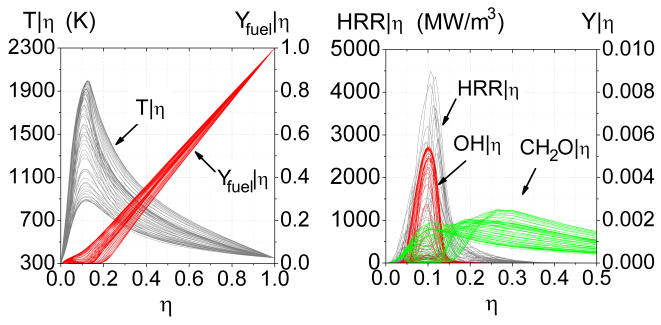

Figure 9: Time evolution of conditional quantities vs. $\eta$ in a CMC cell located at $r / D=$ $0.6, z / D=0.4$.

conditional HRR, $\mathrm{OH}$ mass fraction, and temperature at the stoichiometric mixture fraction (Fig. 8), the flame exhibits sequential extinctions and reignitions. During the extinction, the level of $\mathrm{OH}$ decreases faster than the HRR and even when $\mathrm{OH}$ completely disappears, some residual HRR remains, probably associated with slow oxidation processes (Fig. 8). Peaks of HRR appear during the ignition and extinction transients and the $\mathrm{OHxCH}_{2} \mathrm{O}$ seems to give a good representation of the transient behaviour of the HRR [3]. During the extinction transient, the $\mathrm{OH}$ mass fraction at $\eta_{s t}$ decreases whereas the $\mathrm{CH}_{2} \mathrm{O}$ mass fraction increases. As also pointed out in [8], lower level of $\mathrm{HRR}$ in the region characterized by higher values of $\mathrm{OH}$ corresponds to a fully burning flame at low $\tilde{N}$. The occurrence of local extinction is in general related to the increase of the scalar dissipation rate, however the values are not necessarily higher than the critical value found with the 0D-CMC computation. This suggests that local extinction along the outer flame brush is due to a combination of the effects of micro-mixing and transport in physical space. Occasionally local extinctions also appear in the inner flame region, along the spray path (as indicated by the red arrow in Fig. 7). In this region the local extinction is mainly driven by the scalar dissipation rate with an 


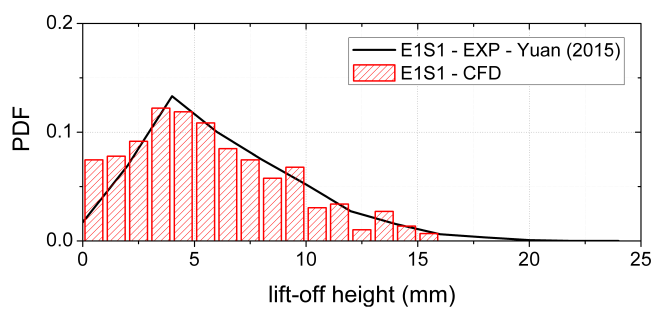

Figure 10: Probability density function of the lift-off height from the experiment 2] and the LES.

important contribution coming from the spray evaporation which, as already observed, has a great effect on the local value of $\widetilde{\xi^{\prime \prime 2}}$ and therefore $\widetilde{N}_{s g s}$.

In order to further validate the capability of the LES/CMC approach to capture the degree of local extinction, the probability density function of the outer flame brush lift-off height was compared with the experiment [2]. Comparisons are shown in Fig. 10. Following the experimental procedure, the lift-off height was determined as the axial distance from the bluff-body surface at which a non-negligible value (higher than $10^{-5}$ ) of $\mathrm{OH}$ mass fraction first appears along the outer flame brush in a $z-r$ cross-section of the flow. Two values, corresponding to the two outer brushes of the flame, of lift-off height were taken from each snapshot, as highlighted in Fig. 7. Images were sampled over $20 \mathrm{~ms}$ with a sampling frequency of $5 \mathrm{kHz}$. The simulation is in very good agreement with the experiment, demonstrating that the method is able to give quantitative predictions of the flame detachment and reattachment to the bluff body. Some small deviations appear for the low values of lift-off height, where the simulation predicts a slightly higher probability of flame reattachment leading to a slightly smaller mean value of the lift-off height. This could be caused by the absence of turbulent fluctuations at the inlet boundary, which would tend to under-estimate extinction 
there. The experimental data have not been analysed from the perspective of quantification of local extinction statistics along the inner flame brush and therefore a quantitative analysis for the inner reacting region is not reported here.

\section{Conclusions}

A swirling ethanol spray flame in conditions close to blow-off showing a large degree of local extinction was investigated using the LES/CMC approach and a detailed chemical mechanism with the main objective to further assess and validate the capability of LES/CMC to capture finite-rate kinetics in turbulent spray flames.

The numerical results agree reasonably well with the experiment in terms of spray location, velocity and size of the droplets, and both instantaneous and mean flame shape. The local extinction along the outer flame brush was found to be influenced by both diffusion in mixture fraction space and transport in physical space. The degree of local extinction in this region was captured well, as demonstrated by comparisons with the experiment in terms of lift-off height statistics. The prediction of the probability density function of the lift-off height is considered a very challenging metric for combustion CFD validation and the results obtained in this work give a further validation of the capability of the LES/CMC approach to capture local extinction. A strong influence of spray evaporation on the sub-grid scale fluctuations of the mixture fraction was observed in the inner flame region where the spray source terms give an important contribution to the sub-grid mixture fraction variance. The effect of spray evaporation on the small-scale gradients has 
a direct impact on the sub-grid scale scalar dissipation rate which in turn affects the local extinction behaviour and further work is necessary to assess the consistency of the usual closures for the scalar dissipation rate and its conditional values when an evaporating spray is present.

\section{Acknowledgements}

This work received funding from Rolls-Royce plc.

\section{References}

[1] D. E. Cavaliere, J. Kariuki, E. Mastorakos, Flow Turbul. Combust. 91 (2013) 347-372.

[2] R. Yuan, Measurements in Swirl-stabilised Spray Flames at Blow-off, Ph.D. thesis, University of Cambridge, 2015.

[3] R. Yuan, J. Kariuki, A. Dowlut, R. Balachandran, E. Mastorakos, Proc. Combust. Inst. 35 (2015) $1649-1656$.

[4] M. Ihme, H. Pitsch, Combust. Flame 155 (2008) 90 - 107.

[5] W. Jones, V. Prasad, Combust. Flame 157 (2010) 1621 - 1636.

[6] A. Garmory, E. Mastorakos, Proc. Combust. Inst. 33 (2011) 1673 - 1680.

[7] V. N. Prasad, M. Juddoo, A. R. Masri, W. P. Jones, K. H. Luo, Combust. Theor. Model. 17 (2013) 483-503.

[8] H. Zhang, A. Garmory, D. E. Cavaliere, E. Mastorakos, Proc. Combust. Inst. 35 (2015) $1167-1174$.

[9] H. Zhang, E. Mastorakos, to appear in Flow Turbul. Combust. (2015).

[10] A. Tyliszczak, D. E. Cavaliere, E. Mastorakos, Flow Turbul. Combust. 92 (2014) $237-267$.

[11] A. Giusti, E. Mastorakos, submitted to Flow Turb. Combust. (2015).

[12] E. Fernández-Tarrazo, A. L. Sánchez, A. Liñán, F. A. Williams, Combust. Flame 147 (2006) $32-38$.

[13] C. Pantano, Journal of Fluid Mechanics 514 (2004) 231-270.

[14] D. O. Lignell, J. H. Chen, H. A. Schmutz, Combust. Flame 158 (2011) 949 - 963. 
[15] P. Jenny, D. Roekaerts, N. Beishuizen, Prog. Energy Combust. Sci. 38 (2012) 846 887.

[16] J. Réveillon, L. Vervisch, Combust. Flame 121 (2000) 75 - 90.

[17] C. Pera, J. Rèveillon, L. Vervisch, P. Domingo, Combust. Flame 146 (2006) 635 648.

[18] S. Ukai, A. Kronenburg, O. Stein, Proc. Combust. Inst. 34 (2013) 1643 - 1650.

[19] S. Ukai, A. Kronenburg, O. Stein, Proc. Combust. Inst. 35 (2015) $1667-1674$.

[20] A. Y. Klimenko, R. W. Bilger, Prog. Energy Combust. Sci. 25 (1999) 595 - 687.

[21] M. Mortensen, R. W. Bilger, Combust. Flame 156 (2009) $62-72$.

[22] A. Garmory, E. Mastorakos, Proc. Combust. Inst. 35 (2015) 1207 - 1214.

[23] A. Triantafyllidis, E. Mastorakos, Flow Turbul. Combust. 84 (2010) 481-512.

[24] S. Navarro-Martinez, A. Kronenburg, F. Di Mare, Flow Turbul. Combust. 75 (2005) 245-274.

[25] E. E. O’Brien, T. Jiang, Phys. Fluids A 3 (1991).

[26] S. Sreedhara, K. Y. Huh, Proc. Combust. Inst. 31 (2007) 2335 - 2342.

[27] G. Borghesi, E. Mastorakos, C. B. Devaud, R. W. Bilger, Combust. Theor. Model. 15 (2011) 725-752.

[28] N. M. Marinov, Int. J. Chem. Kinet. 31 (1999) 183-220.

[29] A. W. Vreman, Phys. Fluids 16 (2004) 3670-3681.

[30] B. Abramzon, W. Sirignano, Int. J. Heat Mass Transfer 32 (1989) 1605 - 1618.

[31] M. Anand, R. Eggels, M. Staufer, M. Zedda, J. Zhu, Proceeding of the ASME Gas Turbine India Conference (2013).

[32] P. N. Brown, A. C. Hindmarsh, Applied Mathematics and Computation 31 (1989) 40 -91 .

[33] S. Ukai, A. Kronenburg, O. T. Stein, Flow Turbul. Combust. 93 (2014) 405-423. 


\section{List of Figures}

Figure 1. Temperature and species mass fractions from a 0D-CMC computation $\left(N_{0}=1201 / \mathrm{s}\right)$.

Figure 2. Mean Mie scattering image from the experiment [2] (left) and the mean Mie equivalent quantity from LES (right) in log-scale.

Figure 3. Numerical results and experimental measurements [2] of droplet mean axial velocity and Sauter Mean Diameter (SMD).

Figure 4. Top row: experimental inverse Abel-transformed time-averaged $\mathrm{OH}^{*}$ [2] (left), LES mean heat release rate (right). Bottom row: mean OH-PLIF from the experiment [2] (left), mean $\mathrm{OH}$ mass fraction from LES (right).

Figure 5. Instantaneous unconditional quantities in a $z-r$ cross section. White line: stoichiometric mixture fraction; red line: $\eta=0.2$; green line: $\eta=$ 0.05 .

Figure 6. Stoichiometric mixture fraction iso-surface coloured by the indicated quantity.

Figure 7. Comparison between an instantaneous OH-PLIF image from the experiment 2] and instantaneous $\mathrm{OH}$ mass fraction from LES. Green arrows: local extinction along the outer flame brush; red arrow: local extinction in the inner flame region.

Figure 8. Time evolution of conditional quantities at the stoichiometric mixture fraction in a CMC cell located at $r / D=0.6, z / D=0.4$.

Figure 9. Time evolution of conditional quantities vs. $\eta$ in a CMC cell located at $r / D=0.6, z / D=0.4$.

Figure 10. Probability density function of the lift-off height from the experiment [2] and the LES. 